\title{
Numerical Renormalization Group Study of the Anderson-Holstein Impurity Model.
}

\author{
A.C. Hewson and D. Meyer \\ Dept. of Mathematics, Imperial College, London SW7 2BZ.
}

\begin{abstract}
We present numerical renormalization group (NRG) calculations for a single-impurity Anderson model with a linear coupling to a local phonon mode. We calculate dynamical response functions, spectral densities, dynamic charge and spin susceptibilities. Being non-perturbative, the NRG is applicable for all parameter regimes. Our calculations cover both weak and strong electron-phonon coupling for zero and finite electron-electron interaction. We interpret the high- and low-energy features and compare our results to atomic limit calculations and perturbation theory. In certain restricted parameter regimes for strong electron-phonon coupling, a soft phonon mode develops inducing a very narrow resonance at the Fermi level.
\end{abstract}

\section{Introduction}

The effects of lattice vibrations on the electronic properties of metals is a well studied branch of condensed matter physics. These effects include specific heat enhancements, strong thermal contributions to the electrical resistivity, and in some materials, superconductivity at low temperatures. There remain, however, some outstanding problems in the field. For example, the extensive studies of small polaron formation, based on the model from the pioneering work of Holstein [1], have given a very precise understanding of systems with one or two electrons present, but there are no comparable results for systems with a finite electron density. There are also many strongly correlated electron systems, such as heavy fermions or valence fluctuations systems, where there is known to be strong coupling between the electrons and the lattice vibrations, in addition to the strong inter-electron interactions. The interplay of phonon and electron effects in these systems has not received much attention. The reason for the lack of progress with these problems is due to the fact there are no reliable calculational techniques in the strong coupling regime, where standard perturbational methods break down. Some non-perturbational techniques have been developed and successfully applied to strongly correlated impurity models but in general these cannot be extended to models of lattice systems. However, with the introduction of dynamical mean field theory (DMFT) [2], it has proved possible to extend some of these non-perturbative techniques to a wide class of lattice models. This approach exploits the fact that certain infinite dimensional lattice models can be mapped onto effective impurity models, together with 
a self-consistency condition [2]. The calculations for the effective impurity models can be carried out using an appropriate non-perturbative method and iterated until selfconsistency is achieved. Though the method is exact in the large dimensionality limit for an appropriately scaled model [3], the method can be applied to a much wider class of models and there is evidence that it provides a very good first order approximation for three dimensional systems. (It clearly breaks down for one-dimensional systems but one-dimensional systems are a class apart.)

Not all techniques for solving strongly correlated impurity models can be used for the effective impurity models generated through the application of DMFT. The methods must be able to handle the dynamical properties of the impurity models, which rules out techniques such as the Bethe ansatz. The method which has been used most extensively for this type of calculation is the quantum Monte Carlo method (QMC). This approach, however, does have the drawback of not being capable of being applied to the very low temperature regime. A method which can be used at $T=0$ is the numerical renormalization group approach (NRG). As developed originally by Wilson for the Kondo model [4, 5, 6] it was used only for the calculation of thermodynamic quantities but the approach was extended later to the calculation of the dynamic properties of impurity models [7, 8], which is required to be able to use the method in conjunction with DMFT for lattice models. The DMFT approach together with the NRG has been applied to the half-filled Hubbard model to study the metal-insulator transition at $T=0$ [9, 10], and these calculations have recently been extended to finite temperatures [11]. Our goal is to extend this approach to include the effects of coupling to local lattice modes, and address some of the outstanding questions concerning the interplay of strong correlation and strong electron-phonon interactions. The results should provide some insights into the physics of systems such as heavy fermions, valence fluctuators, manganites, and the cuprate high temperature superconductors.

In this paper we perform NRG calculations solely for an impurity model with a coupling to a local phonon mode. This is a necessary first step to the eventual aim of incorporating the self-consistency constraint of the DMFT and the application to models for lattice systems. We introduce the impurity model in the next section.

\section{The Anderson-Holstein Impurity Model}

The model we study is a single impurity Anderson model [12 with a linear coupling to a local phonon mode, as in the the Holstein model [1]. We will refer to this model as the Anderson-Holstein (A-H) model. The corresponding Hamiltonian for the model is

$$
\begin{aligned}
H= & \sum_{\sigma} \epsilon_{f} f_{\sigma}^{\dagger} f_{\sigma}+U f_{\sigma}^{\dagger} f_{\sigma} f_{\bar{\sigma}}^{\dagger} f_{\bar{\sigma}}+\lambda\left(b^{\dagger}+b\right)\left(\sum_{\sigma} f_{\sigma}^{\dagger} f_{\sigma}-1\right) \\
& +\sum_{\mathbf{k}, \sigma} V_{\mathbf{k}}\left(f_{\sigma}^{\dagger} c_{\mathbf{k} \sigma}+c_{\mathbf{k} \sigma}^{\dagger} f_{\sigma}\right)+\sum_{\mathbf{k} \sigma} \epsilon_{\mathbf{k}} c_{\mathbf{k} \sigma}^{\dagger} c_{\mathbf{k} \sigma}+\omega_{0} b^{\dagger} b
\end{aligned}
$$

which describes an impurity d level $\epsilon_{d}$, hybridized with conduction electrons of the host metal via a matrix element $V_{k}$, with an interaction term $U$ between the electrons in the 
localized $\mathrm{f}$ ( or d) state, where $n_{f, \sigma}=f_{\sigma}^{\dagger} f_{\sigma}$ is the occupation number of this state which is linearly coupled to a local oscillator of frequency $\omega_{0}$.

A very similar model which does not have the interaction term $U$, but does not quite correspond to the A-H model with $U=0$ as the electrons are spinless, has been studied previously [13, 14. Both the model with spin and the spin-less model can be solved exactly in the case of one electron only in the system 13. As the interaction $U$ does not come into play in this situation the results of both models are essentially equivalent. However, there is no exact solution for the many-electron case even for the spin-less model and $U=0$.

We will solve the model (1) using the numerical renormalization group method. We will briefly outline the general NRG scheme which is applicable to any impurity model with local interactions and coupling to local bosonic or fermionic fields [5, 6]. The conduction band of the model is logarithmically discretized and mapped onto a semiinfinite linear chain coupled to the impurity at one end such that the hopping matrix elements along the chain fall off exponentially $\left(\sim \Lambda^{-n / 2}\right)$. The many-body states and energy levels are calculated iteratively starting from the impurity adding an extra site of the chain in each step. After about 5-6 steps, the number of states has to be truncated to the 300-800 lowest ones depending on the application in each step. This method has been generalized to the calculation of dynamic as well as static properties [7, 8]. In applying this approach to the model with phonons (Eq. (1)), a truncation of higher-energy steps is already necessary at the initial step. The probability distribution function of finding $n$ excited phonons in the system has a clear maximum around $n=\bar{n}=v / \omega_{0}$, as can be read off equation (15), and falls off rapidly for larger values of $n$. So it is sufficient to keep only states with $n<n_{\text {cutoff }}$ where we have chosen an initial $n_{\text {cutoff }}=4 \bar{n}$. We have used the discretization parameter $1.7<\Lambda<2$., and kept at least 600 states in each iteration (see Ref. [5, 11]).

To calculate the spectral function on the real axis, we need the Fourier transform of the double-time one-electron Green's function for the local d-electron. We use this in the standard form,

$$
G_{\sigma}(\omega)=\frac{1}{\omega-\epsilon_{\mathrm{f}}+i \Delta-\Sigma_{\sigma}(\omega)},
$$

where we have taken the hybridization function $\Delta(\omega)=\pi \sum_{k}\left|V_{k}\right|^{2} \delta\left(\omega-\epsilon_{k}\right)$ to be independent of $\omega$, corresponding to wide conduction band with a flat density of states. The self-energy $\Sigma_{\sigma}(\omega)$ will be calculated, as in reference [15], from the ratio of two Green's functions. Due to the additional interaction term with the phonon there are two contributions so that $\Sigma_{\sigma}(\omega)=\Sigma_{\sigma}^{U}(\omega)+\Sigma_{\sigma}^{\lambda}(\omega)$, with

$$
\Sigma_{\sigma}^{U}(\omega)=U \frac{F_{\sigma}(\omega)}{G_{\sigma}(\omega)}, \quad \Sigma_{\sigma}^{\lambda}(\omega)=\lambda \frac{M_{\sigma}(\omega)}{G_{\sigma}(\omega)},
$$

where $F_{\sigma}(\omega)$ and $M_{\sigma}(\omega)$ are the higher order Green's functions,

$$
F_{\sigma}(\omega)=\left\langle\left\langle f_{\sigma} f_{\bar{\sigma}}^{\dagger} f_{\bar{\sigma}}, f_{\sigma}^{\dagger}\right\rangle\right\rangle_{\omega}, \quad M_{\sigma}(\omega)=\left\langle\left\langle f_{\sigma}\left(b^{\dagger}+b\right), f_{\sigma}^{\dagger}\right\rangle\right\rangle_{\omega} .
$$


Details of the derivation of this result from the equations of motion are given in the Appendix A. In Appendix B, more details regarding the calculation of $M_{\sigma}(\omega)$ are presented.

We will also calculate the spectral density of the Green's function of the phonon mode, $D(\omega)=\left\langle\left\langle\left(b^{\dagger}+b\right),\left(b^{\dagger}+b\right)\right\rangle\right\rangle_{\omega}$. This can be expressed in terms of the local charge susceptibility,

$$
D(\omega)=D^{0}(\omega)+\lambda^{2} D^{0}(\omega)\langle\langle\hat{O}, \hat{O}\rangle\rangle_{\omega} D^{0}(\omega),
$$

where

$$
D^{0}(\omega)=\frac{2 \omega_{0}}{\omega^{2}-\omega_{0}^{2}}
$$

is the non-interacting phonon propagator, and $\langle\langle\hat{O}, \hat{O}\rangle\rangle_{\omega}$ with $\hat{O}=\sum_{\sigma} f_{\sigma}^{\dagger} f_{\sigma}-1$ is the local dynamic charge susceptibility.

It will be useful in interpreting the numerical results to compare them with results from perturbation theory in the relevant regimes. We will set up the general perturbational approach in this section and give specific results later. Starting from expression for the partition function $Z$ in the form,

$$
Z / Z_{0}=\left\langle T e^{-\int_{0}^{\beta} H_{\text {int }}(\tau) d \tau}\right\rangle_{0},
$$

where $T$ is this usual time-ordering operator and $H_{\text {int }}(\tau)$ is the interaction part of the Hamiltonian (including the hybridization term) in the interaction representation with $\tau$ as the imaginary time variable and $\beta=1 / T$, and \langle\rangle$_{0}$ denotes a thermal expectation value with respect to the non-interacting part of the Hamiltonian. We can remove explicitly the phonon operators and rewrite (17) as

$$
Z / Z_{0}=\left\langle T e^{-\int_{0}^{\beta}\left(H_{\mathrm{int}}^{F}(\tau)+H_{\mathrm{ret}}(\tau)\right) d \tau}\right\rangle_{0},
$$

where $H_{\text {int }}^{F}$ is the purely fermionic interaction part of the Hamiltonian (11) and $H_{\text {ret }}$ is an additional retarded local fermionic interaction due to the elimination of the phonon terms, which is given by

$$
H_{\mathrm{ret}}(\tau)=\lambda^{2} \int_{0}^{\tau} \hat{O}(\tau) \hat{O}\left(\tau^{\prime}\right) D^{0}\left(\tau-\tau^{\prime}\right) d \tau^{\prime},
$$

where $D^{0}(\tau)$ is the non-interacting phonon Green's function in the imaginary time form. Details of this derivation are given in Appendix C.

It will be convenient to convert this expression to a path integral over fermionic Grassmann variables and integrate out the conduction electrons. We can then write (7) in the form,

$$
Z=\int \prod_{\sigma} \mathcal{D}\left(\bar{f}_{\sigma}\right) \mathcal{D}\left(f_{\sigma}\right) e^{-\int_{0}^{\beta} \mathcal{L}_{\text {eff }}(\tau) d \tau},
$$

where

$$
\begin{aligned}
\mathcal{L}_{\mathrm{eff}}(\tau)= & \int_{0}^{\beta} d \tau^{\prime} \sum_{\sigma} \bar{f}_{\sigma}(\tau)\left[G_{\sigma}^{(0)}\left(\tau-\tau^{\prime}\right)\right]^{-1} f_{\sigma}\left(\tau^{\prime}\right)+U n_{\uparrow}(\tau) n_{\downarrow}(\tau) \\
& +\frac{\lambda^{2}}{2} \int_{0}^{\beta} d \tau^{\prime} D^{0}\left(\tau-\tau^{\prime}\right)\left(\sum_{\sigma} n_{\sigma}(\tau)-1\right)\left(\sum_{\sigma^{\prime}} n_{\sigma^{\prime}}\left(\tau^{\prime}\right)-1\right)
\end{aligned}
$$


where $\bar{f}_{\sigma}(\tau), f_{\sigma}(\tau)$ are Grassmann variables, $n_{\sigma}(\tau)=\bar{f}_{\sigma}(\tau) f_{\sigma}(\tau)$, and $G_{\sigma}^{(0)}(\tau)$ is the imaginary-time of the non-interacting one electron Green's function for the localized f-electrons. By introducing a Grassmann source field for the f-electrons one can convert this partition function into a generating function for the interacting local Green's function $G_{\sigma}(\tau)$ in the standard way [16]. This gives a convenient way of generating the perturbation theory and Feynman diagrams for this Green's function.

In the limit $\omega_{0} \rightarrow \infty$ such that $v=\lambda^{2} / \omega_{0}$ remains finite the effective interaction becomes an instantaneous one equal to $-v\left(\sum_{\sigma} n_{\sigma}-1\right)^{2}$. This interaction term can be absorbed into the standard Anderson model with the terms linear in $n_{\sigma}$ being absorbed by modifying $\epsilon_{f} \rightarrow \epsilon_{f}+v$, and the remaining part by changing $U$ to $U-2 v$. In this way of taking the limit $\omega_{0} \rightarrow \infty$, the ratio $v / \omega_{0} \rightarrow 0$. But there is also a high frequency regime of interest in which both $\omega_{0}$ and $v$ are large such that $v / \omega_{0} \sim 1$. For this parameter range it is convenient to apply the canonical transformation $\hat{U}^{-1} H \hat{U}$ with $\hat{U}$ given by

$$
\hat{U}=e^{-\frac{\lambda}{\omega_{0}}\left(b^{\dagger}-b\right)\left(\sum_{\sigma} n_{\sigma}-1\right)}
$$

which is a displaced oscillator transformation such that

$$
\begin{aligned}
\tilde{b}=\hat{U}^{-1} b \hat{U}=b & -\frac{\lambda}{\omega_{0}}\left(\sum_{\sigma} n_{\sigma}-1\right), \text { and } \tilde{f}_{\sigma}=\hat{U}^{-1} f_{\sigma} \hat{U}=e^{-\frac{\lambda}{\omega_{0}}\left(b^{\dagger}-b\right)} f_{\sigma} . \\
H^{\prime}=\hat{U}^{-1} H \hat{U}= & \sum_{\sigma} \bar{\epsilon}_{f} f_{\sigma}^{\dagger} f_{\sigma}+\bar{U} f_{\sigma}^{\dagger} f_{\sigma} f_{\bar{\sigma}}^{\dagger} f_{\bar{\sigma}} \\
& +\sum_{\mathbf{k}, \sigma} V_{\mathbf{k}}\left(e^{\frac{\lambda}{\omega_{0}}\left(b^{\dagger}-b\right)} f_{\sigma}^{\dagger} c_{\mathbf{k} \sigma}+e^{-\frac{\lambda}{\omega_{0}}\left(b^{\dagger}-b\right)} c_{\mathbf{k} \sigma}^{\dagger} f_{\sigma}\right)+\sum_{\mathbf{k} \sigma} \epsilon_{\mathbf{k}} c_{\mathbf{k} \sigma}^{\dagger} c_{\mathbf{k} \sigma}+\omega_{0} b^{\dagger} b,
\end{aligned}
$$

where $\bar{\epsilon}_{f}=\epsilon_{f}+v$ and $\bar{U}=U-2 v$. The exponential term in the phonon operators, which multiplies the hybridization term, arises because a cloud of phonons may be created or absorbed when the f-electron occupation changes, due to the coupling to the lattice. If we take the expectation value of this Hamiltonian in the zero phonon state $|0\rangle$ then, as $\left\langle 0\left|e^{ \pm \lambda / \omega_{0}\left(b^{\dagger}-b\right)}\right| 0\right\rangle=e^{-v / 2 \omega_{0}}$, the effective resonance width becomes $\bar{\Delta}=e^{-v / \omega_{0}} \Delta$. For $v / \omega_{0} \approx 2$ or 3 , this would amount to a significant narrowing of the resonance for the non-interacting model. This exponential renormalization factor is a common feature of strongly coupled electron-phonon problems but, as was shown in earlier work of Hewson and Newns [13, 14] for the spinless model, this resonance narrowing does not in general occur in the strong coupling regime for this model, but only in a certain parameter regime.

In the zero hybridization limit for the transformed Hamiltonian in equation (14) the electrons and phonons become decoupled. However in this limit the calculation of the Green's function in terms of the original f-electron operators is not trivial, as it involves the unitary transformation $\hat{U}$ but it can be evaluated exactly by using the well known techniques for calculating the expectation values of exponentials with a linear 
combinations of bose creation and annihilation operators [17]. The result for $T=0$ is

$$
\begin{aligned}
G_{\sigma}(\omega)=e^{-v / \omega_{0}} \sum_{n=0}^{n=\infty} \frac{\left(v / \omega_{0}\right)^{n}}{n !}[ & \frac{\left\langle\left(1-n_{f, \sigma}\right)\left(1-n_{f,-\sigma}\right)\right\rangle}{\omega-\bar{\epsilon}_{f}-n \omega_{0}}+\frac{\left\langle\left(1-n_{f, \sigma}\right) n_{f,-\sigma}\right\rangle}{\omega-\bar{\epsilon}_{f}-\bar{U}-n \omega_{0}} \\
& \left.+\frac{\left\langle n_{f, \sigma}\left(1-n_{f,-\sigma}\right)\right\rangle}{\omega-\bar{\epsilon}_{f}+n \omega_{0}}+\frac{\left\langle n_{f, \sigma} n_{f,-\sigma}\right\rangle}{\omega-\bar{\epsilon}_{f}-\bar{U}+n \omega_{0}}\right],
\end{aligned}
$$

where the expectation values, such as $\left\langle\left(1-n_{f, \sigma}\right)\left(1-n_{f,-\sigma}\right)\right\rangle$, are calculated using (14) in the 'atomic' limit $V_{k}=0$ for $T=0$.

In the weak coupling regime we can use perturbation theory based on the original form of the model given in equation (1). Using the finite temperature perturbation expansion for the self-energy $\Sigma_{\sigma}^{\lambda}(\omega)$, carried out to order $\lambda^{2}$, and analytically continued to real frequency $\omega$, we obtain

$$
\begin{aligned}
\Sigma_{\sigma}^{\lambda(2)}(\omega)= & \frac{2 \lambda^{2}}{\omega_{0}}\left(1-\sum_{\sigma} n_{f, \sigma}\right) \\
& +\lambda^{2} \int \rho_{f, \sigma}(\epsilon)\left\{\frac{f(\epsilon)+n_{\mathrm{b}}\left(\omega_{0}\right)}{\omega-\epsilon+\omega_{0}}+\frac{1-f(\epsilon)+n_{\mathrm{b}}\left(\omega_{0}\right)}{\omega-\epsilon-\omega_{0}}\right\} d \epsilon,
\end{aligned}
$$

where $n_{\mathrm{b}}(\epsilon)$ and $f(\epsilon)$ are the Bose-Einstein and Fermi-Dirac distribution functions. The occupation value $n_{f, \sigma}$ of the f-state with spin $\sigma$, and the corresponding spectral density $\rho_{f, \sigma}(\omega)$, can in principle include self-energy contributions from the local interaction term $U$. These quantities to zero order in $U$ are given by

$$
n_{f, \sigma}^{0}=\frac{1}{2}-\frac{1}{\pi} \tan ^{-1}\left(\frac{\epsilon_{f}}{\Delta}\right), \quad \rho_{f, \sigma}^{0}(\epsilon)=\frac{\Delta / \pi}{\left(\epsilon-\epsilon_{f}\right)^{2}+\Delta^{2}} .
$$

Substituting these expressions into (16) for the self-energy at $T=0$, we find

$$
\begin{gathered}
\operatorname{Re} \Sigma_{\sigma}^{\lambda(2)}(\omega)=\frac{4 \lambda^{2}}{\omega_{0} \pi} \tan ^{-1}\left(\frac{\epsilon_{f}}{\Delta}\right)+ \\
\frac{\lambda^{2}}{\pi} \operatorname{Im}\left\{\ln \left(\frac{\epsilon_{f}+i \Delta}{\left|\omega+\omega_{0}\right|}\right) \frac{1}{\omega-\epsilon_{f}+\omega_{0}-i \Delta}-\ln \left(\frac{\epsilon_{f}+i \Delta}{\left|\omega-\omega_{0}\right|}\right) \frac{1}{\omega-\epsilon_{f}-\omega_{0}-i \Delta}\right\}
\end{gathered}
$$

and

$\operatorname{Im} \Sigma_{\sigma}^{\lambda(2)}(\omega)=-\pi \lambda\left(\left(1-\Theta\left(\omega+\omega_{0}\right)\right) \frac{\Delta}{\left(\omega+\omega_{0}-\epsilon_{f}\right)^{2}+\Delta^{2}}+\Theta\left(\omega-\omega_{0}\right) \frac{\Delta}{\left(\omega-\omega_{0}+\epsilon_{f}\right)^{2}+\Delta^{2}}\right)$.

The corresponding expression for $\Sigma_{\sigma}^{U}(\omega)$ to order $U^{2}$ is

$\Sigma_{\sigma}^{U(2)}(\omega)=U n_{f,-\sigma}^{(2)}+U^{2} \int \tilde{\rho}_{f, \sigma}(\epsilon) \tilde{\rho}_{f,-\sigma}\left(\epsilon^{\prime}\right) \tilde{\rho}_{f,-\sigma}\left(\epsilon^{\prime \prime}\right) \frac{D\left(\epsilon, \epsilon^{\prime}, \epsilon^{\prime \prime}\right)}{\omega-\epsilon+\epsilon^{\prime}-\epsilon^{\prime \prime}} d \epsilon d \epsilon^{\prime} d \epsilon^{\prime \prime}$

where

$$
D\left(\epsilon, \epsilon^{\prime}, \epsilon^{\prime \prime}\right)=f\left(\epsilon^{\prime}\right)-f\left(\epsilon^{\prime}\right) f\left(\epsilon^{\prime \prime}\right)+f(\epsilon) f\left(\epsilon^{\prime \prime}\right)-f(\epsilon) f\left(\epsilon^{\prime}\right) .
$$

The effects of phonon scattering could be included in the f-spectral density $\rho_{f, \sigma}(\epsilon)$, and $n_{f, \sigma}$, in evaluating (20). If terms of order $\lambda^{2}$ were to be included they would generate corrections of order $\lambda^{2} U^{2}$. These contributions would be distinct from those of the same order generated by including terms of order $U^{2}$ in evaluating $\Sigma^{\lambda}(\omega)$ from equation (16). 
To zero order in $\lambda$ we use (17) in evaluating (20). These results can be used to compare with the NRG ones in the weak coupling regime.

There is a large parameter space for this model so we will present the NRG results in four sections, corresponding to the symmetric and asymmetric models with $U=0$, and then the same models with $U \neq 0$.

\section{Symmetric $U=0$ Model}

In the upper panel of figure 1 1 we present the NRG results for the spectral density of the $\mathrm{f}$-Green's function for various values of $\lambda$. The bare phonon frequency has been taken to be $\omega_{0}=0.05$ in this and all subsequent plots. The significant features are the narrowing and virtual collapse of the central resonance with increasing coupling strength $\lambda$ and the development and outward shift of high energy peaks. This shift is proportional to $\lambda^{2}$ and can be understood by looking at the corresponding results in the atomic limit $(V=0)$. The spectral weights and positions of the atomic-limit excitation peaks of equation (15) for selected values of $\lambda$ are plotted in the middle panel of figure 1. The high-energy features in the upper panel correspond to a broadening those seen in the atomic limit. In this case $\overline{\epsilon_{f}}=v$ and $\bar{U}=-2 v$, so the effective interaction is an attractive one. The peaks below the Fermi level correspond to removing an electron from a doubleoccupied site, the peaks above to adding an electron to a previously unoccupied site. The probability that the impurity is singly-occupied for finite $\lambda$ vanishes exponentially with $\lambda^{2}$ (see equation (15)). The narrowing of the central peak, the zero-phonon peak occurs very rapidly with increase of $\lambda^{2}$. On the basis of equation (14), one might expect the narrowing to follow the exponential form $\sim e^{-v / \omega_{0}}$. In figure 2 we plot the relative width of the central peak as function of $\lambda$. Only for small values of $\lambda$ is it approximately proportional to $\exp \left(-v / \omega_{0}\right)$. It decreases much more rapidly with higher coupling strengths. This could be due to the effect of the softening of the phonon mode.

The imaginary part of the phonon Green's function, displayed in figure 3, shows clear evidence of the emergence of a soft phonon mode already for intermediate coupling strength. The inset shows the same as the large figure, but over a larger frequency range, displaying also the 'bare phonon' peaks at $\omega= \pm 0.05$. With increasing $\lambda$, another peak appears at lower frequency. This soft phonon mode can be traced back to features in the charge susceptibility. Equation (5) shows the relation between the phonon Green's function and the dynamic charge susceptibility. The soft phonon mode reflects the lower energy peak in the charge susceptibility. Figure 4 shows the position of the lower energy

peak in phonon propagator. It falls off approximately with $\omega_{\lambda}=1 / 30 \exp \left(-\frac{(2.45 * \lambda)^{2}}{\omega_{0}^{2}}\right)$. With this result, we also reach a good fit to the data of figure 2 if we assume the zero-phonon peak width to behave like $\exp \left(-v\left(\frac{1}{\omega_{0}}+\frac{0.03}{\omega_{\lambda}}\right)\right.$ instead of $\exp \left(-v / \omega_{0}\right)$.

A comparison of our results with those of lowest-order perturbation theory, equation (16), which is displayed in the lowest panel of figure 1, reveals the limitations of the perturbational result in this parameter regime. We would not expect to get the exponential narrowing of the zero-phonon peak from a weak-coupling result, but 

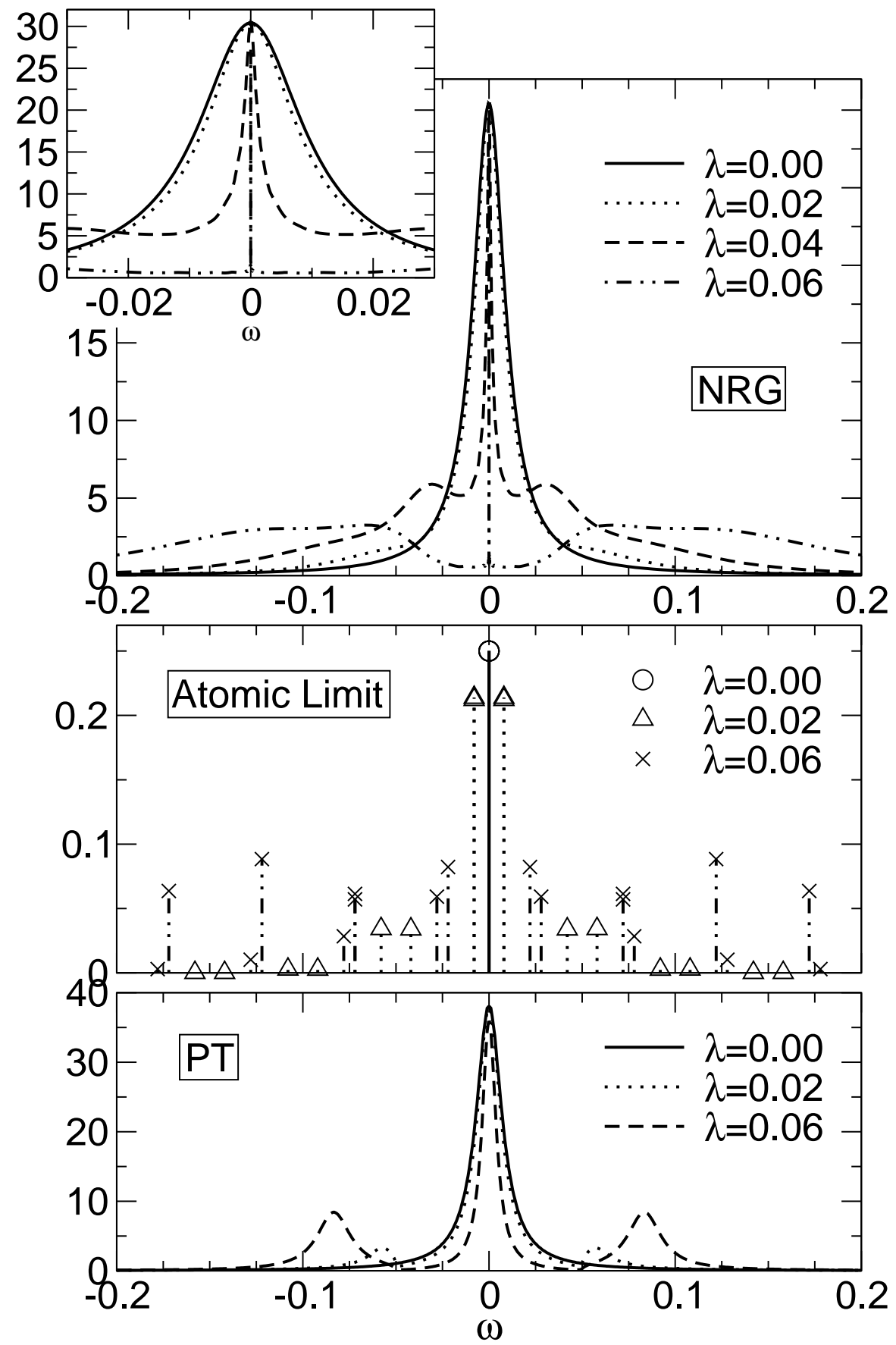

Figure 1. Spectral density $A(\omega)=-\frac{1}{\pi} \Im G_{\sigma}(\omega)$ for $U=0, \epsilon_{f}=0, V=0.1 \rightarrow \Delta \approx$ 0.016 and $\omega_{0}=0.05$. The respective values of $\lambda$ are given in the graph. The upper panel shows NRG results, the two lower panels the atomic limit and the perturbation theory $(\mathrm{PT})$ results. For the atomic limit calculation, the height of the peaks is a measure of the respective spectral weight of the excitation.

the high-energy features are also not well reproduced. Although there are high-energy peaks, their positions are in poor agreement with the NRG results and their shift to higher frequencies is proportional to $\lambda$ instead of $\lambda^{2}$. 


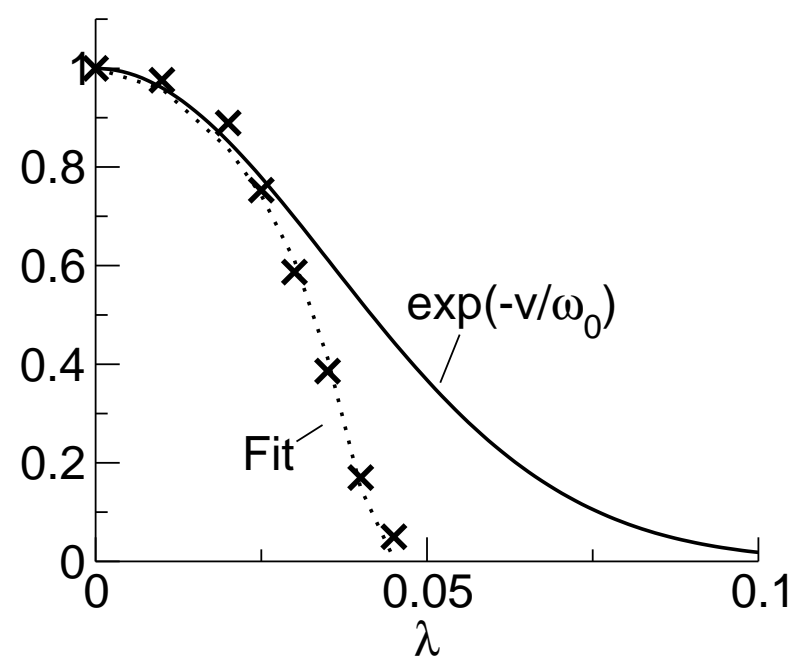

Figure 2. Width of central resonance ('zero-phonon peak') as function of $\lambda$ for the parameters of figure 1 (NRG calculation). The solid and dashed lines are explained in the text.

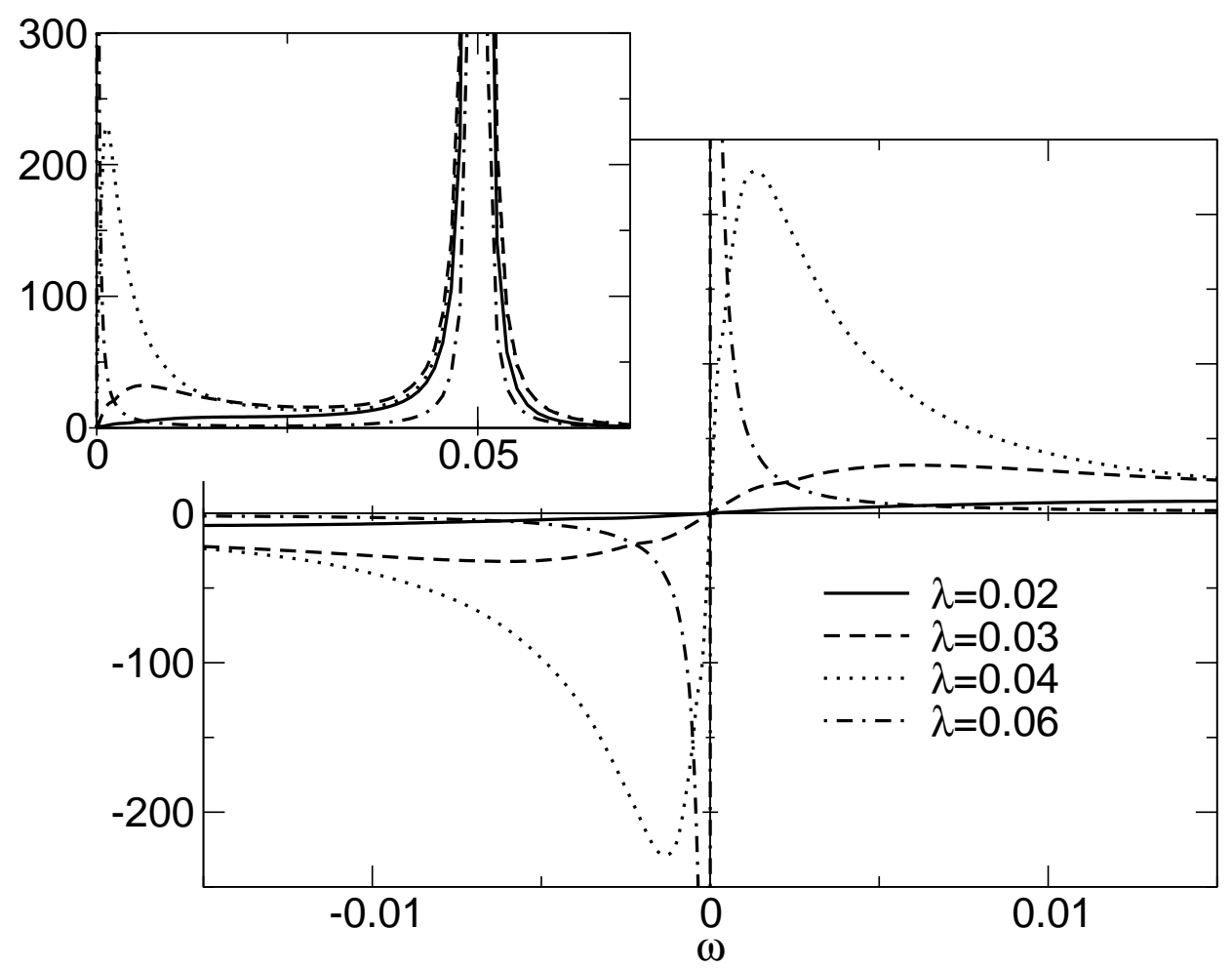

Figure 3. Imaginary part of the phonon propagator for the same parameters as used in figure 1. The inset shows the same quantity on a larger energy scale.

\section{Asymmetric $U=0$ Model}

As soon as we introduce an asymmetry into the model, we find a qualitatively different picture. In figure 5 we plot the NRG, atomic limit and perturbation theory results for 


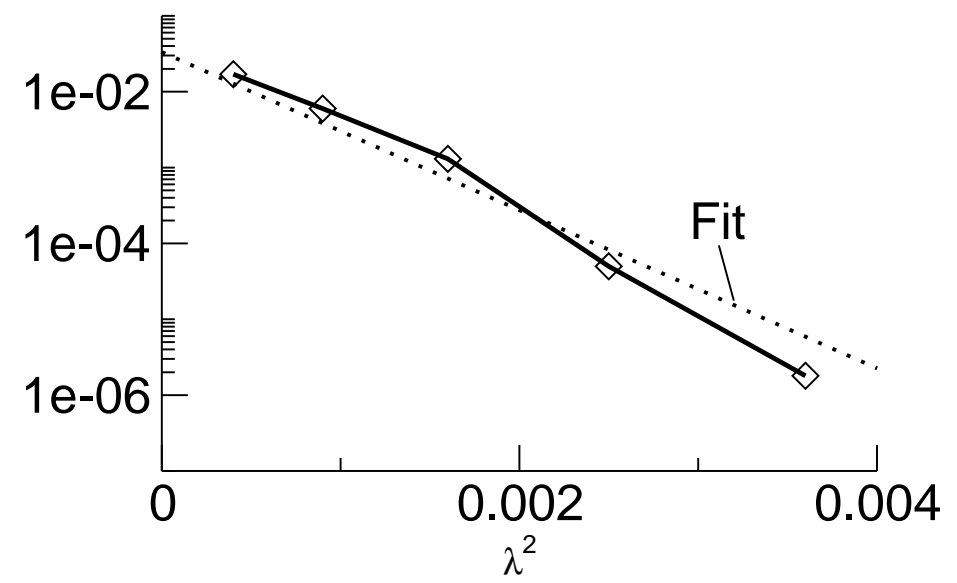

Figure 4. Position of the low-energy peak of the phonon propagator (see figure 3 ) plotted on a logarithmic scale vs. $\lambda^{2}$.

the spectral density of the local Green's function for a range of values of $\lambda$ similar to figure 1, but with an $f$-level located at $\epsilon_{f}=-0.05$. In this spectrum there is a single peak corresponding to an excitation from a doubly-occupied impurity site, positioned at $\overline{\epsilon_{f}}+\bar{U}-\bar{n} \omega_{0}=\epsilon_{f}-v-\bar{n} \omega_{0}$, where $\bar{n}=v / \omega_{0}$ is approximately the mean number of phonons excited. The shift in the peak to lower values reflects that seen in the atomic limit, equation (15). Additionally, the peak broadens with increasing $\lambda$ due to the higher probability of exciting multiple phonons on removing the local electron. As the peak moves away from the Fermi level, the charge fluctuations are suppressed. This is manifest in the absence of the soft phonon mode, as can be seen in figure 6 showing the phonon propagator in this parameter range (cf. equation (5)).

In contrast to the symmetric case, the perturbational result, shown in the lower panel of figure 5, does give quite good agreement of the peak positions, the increase of broadening, however, is absent.

\section{Symmetric $U \neq 0$ Model}

In figure 7, we plot the spectral density for $U=0.1$ and $\epsilon_{f}=-0.05$. In contrast to the $U=0$ case, the central peak initially broadens with increase of $\lambda$ and then narrows rapidly. When $\lambda=0, U / \pi \Delta=2.03$, we are initially in the Kondo regime and so we can interpret the central peak here as Kondo resonance with a width of the order of $2 T_{K} \sim(U \Delta)^{\frac{1}{2}} \exp \left(\frac{-U}{\pi \Delta}\right)$. Hence if $U$ is replaced by $\bar{U}=U-2 v$ as in equation (14) then $T_{K}$ would be expected to increase, provided $\bar{U}$ remains sufficiently large so that we stay in the Kondo regime. The situation will change as $\bar{U}$ decreases below $\pi \Delta$. When $\bar{U}$ becomes negative we expect the results to be similar to the $U=0$ symmetric case considered in Sec. 3. This is qualitatively the case, and a very rapid narrowing of the central peak can be seen in the inset of the top panel in figure 0 for $\lambda>\lambda_{c}$ where $\lambda_{c}=\sqrt{U \omega_{0} / 2}=0.05$. 
$N R G$ study of the Anderson-Holstein model
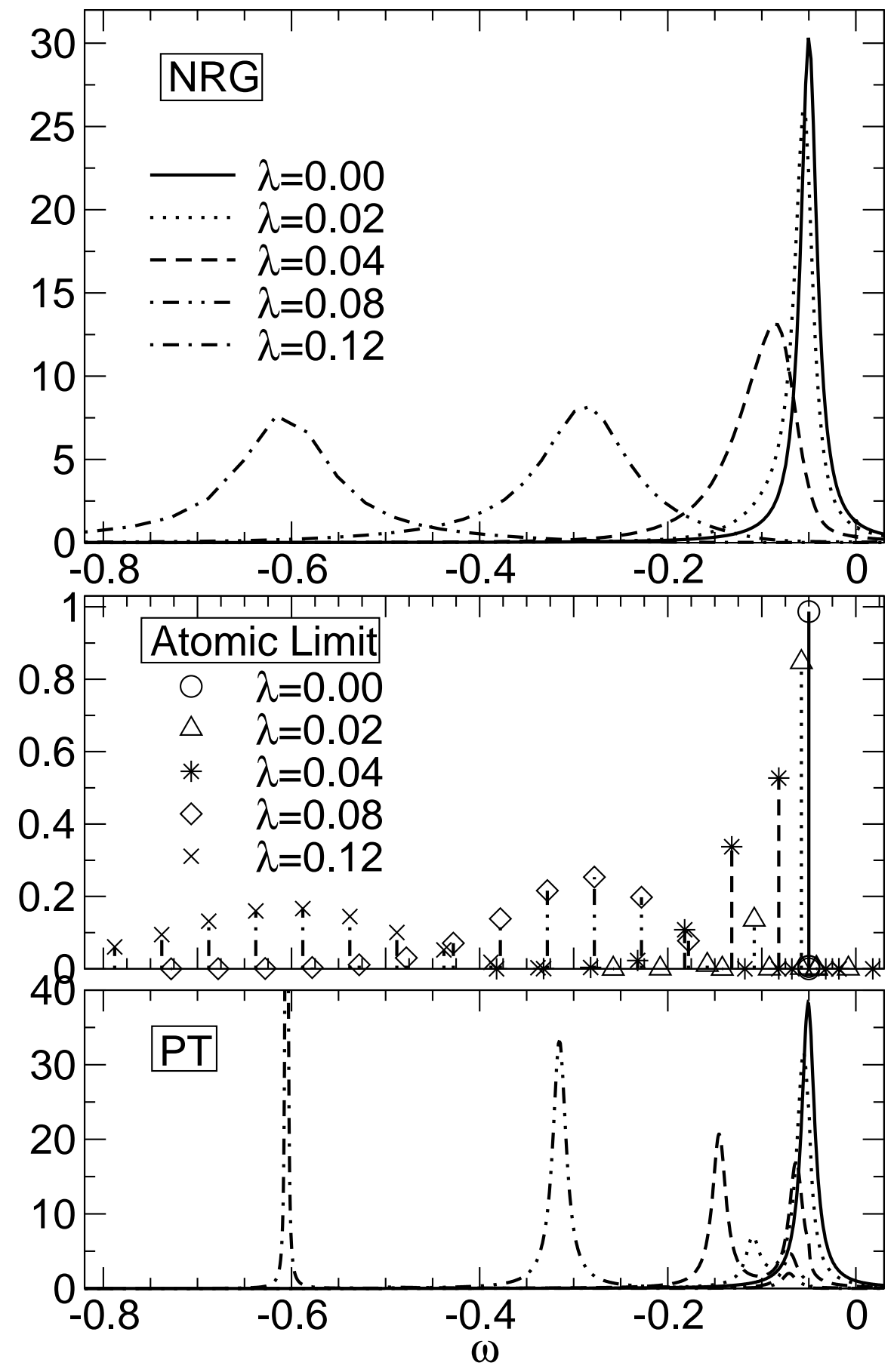

Figure 5. Same as figure 1, but with $\epsilon_{f}=-0.05$.

For small $\lambda$, we see the typical charge excitations of the symmetric Anderson model at $\omega= \pm U / 2$. For larger values of $\lambda$, we see in general four high-energy peaks. The positions and relative weights of these peaks can be understood in terms of the diagram shown in figure 8, where we plot the positions of the dominant excitation peaks taken from the atomic limit result displayed in the middle panel of figure 7 . These are obtained from the atomic limit Green's function by replacing the multi-phonon peaks for each 


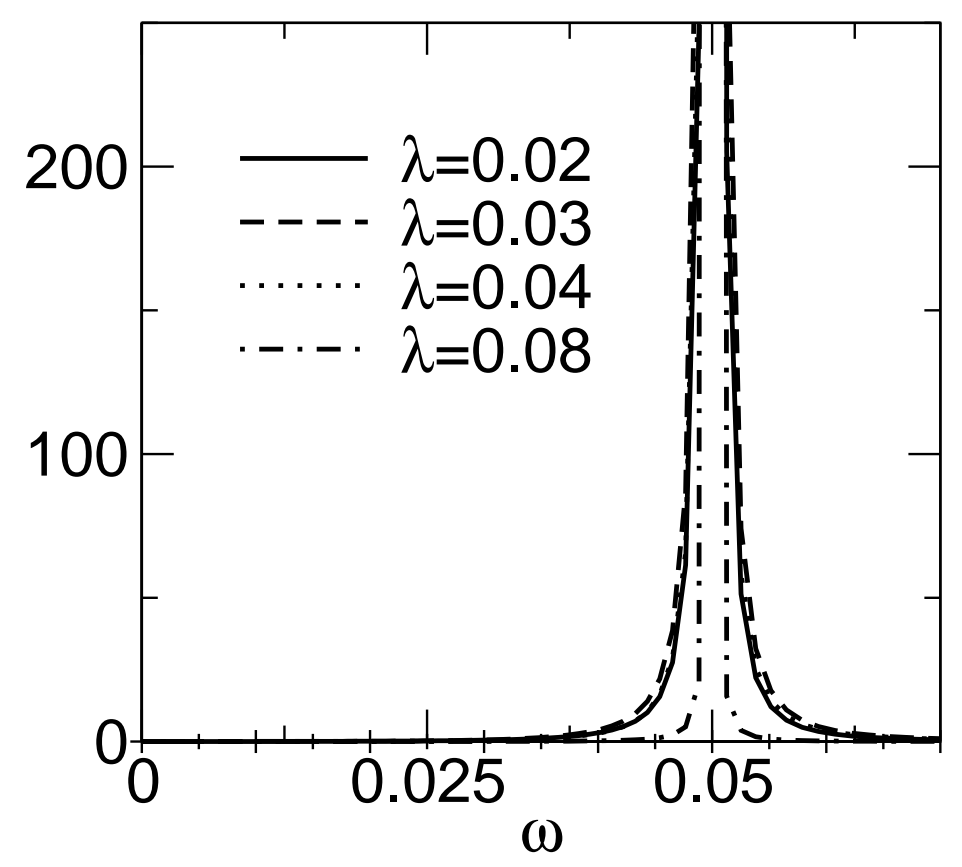

Figure 6. Imaginary part of the phonon propagator for the parameters of figure

term in equation (15) by a single peak with $\bar{n}$ excited phonons:

$$
\begin{aligned}
& \omega=\overline{\epsilon_{f}}+\bar{n} \omega_{0}=\epsilon_{f}+2 v \quad \text { from zero-occupied site } \\
& \omega=\overline{\epsilon_{f}}-\bar{n} \omega_{0}=\epsilon_{f} \quad \text { from single-occupied site } \\
& \omega=\overline{\epsilon_{f}}+\bar{U}+\bar{n} \omega_{0}=\epsilon_{f}+U \quad \text { from single-occupied site } \\
& \omega=\overline{\epsilon_{f}}+\bar{U}-\bar{n} \omega_{0}=\epsilon_{f}+U-2 v \text { from double-occupied site }
\end{aligned}
$$

The total weight in each peak is the sum of the weights of the contributing excitations in the atomic limit. The size of the symbols in each curve indicates the corresponding weight factor. One observes the transfer of weight with increasing $\lambda$ from the excitations in which the site is initially singly-occupied to those of the zero- and doubly-occupied states as $\bar{U}$ changes sign. In the approach to the regime where these levels cross, all four peaks can be clearly distinguished in figure 7 .

The imaginary part of the phonon Green's function is shown in figure 9. We see that while $\bar{U}>0$ charge fluctuations are suppressed and there is no soft phonon mode $\omega_{\lambda}$. This mode only emerges when $\bar{U}$ becomes negative. Its appearance clearly correlates with the collapse of the central peak.

For comparison, the perturbational results corresponding to the lowest-order diagrams in $U$ and $\lambda$ are shown in the lower panel of figure 77. It only gives a good approximation to the NRG results for $\lambda=0$, and there is clear disagreement for finite $\lambda$ as in the $U=0$ case discussed in Sec. 3 . 

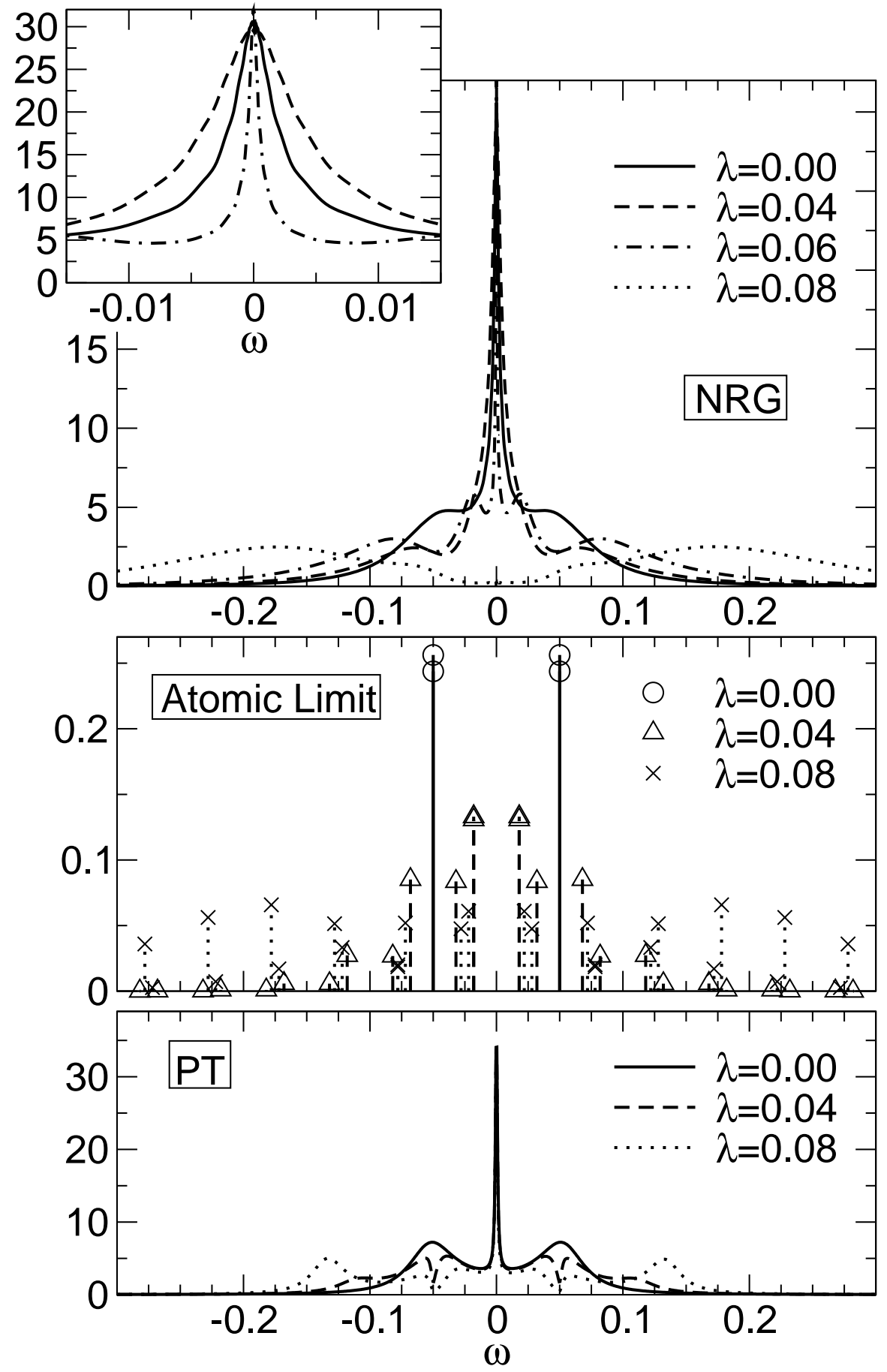

Figure 7. Spectral density for $U=0.1, \epsilon_{f}=-0.05, V=0.1 \rightarrow \Delta \approx 0.016$ and $\omega_{0}=0.05$. The respective values of $\lambda$ are given in the graph. The upper panel shows NRG results, the two lower panels the atomic limit and the perturbation theory (PT) results. For the atomic limit calculation, the height of the peaks is a measure of the respective spectral weight of the excitation.

\section{Asymmetric $U \neq 0$ Model}

In Figs. 10 and 11, we plotted the spectral density for $U=0.2$ and $U=0.05$, respectively $\left(\epsilon_{f}=-0.05, V=0.1 \rightarrow \Delta=0.016, \omega_{0}=0.05\right)$. In the first case, for $\lambda=0$, we are 


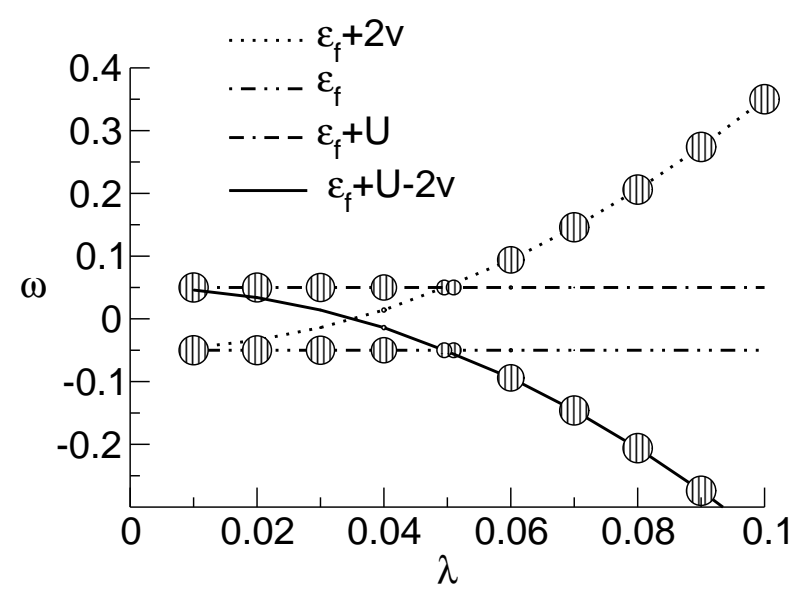

Figure 8. Positions of the dominant peaks for the atomic limit calculation from figure 7. The size of the circles shows the total weight for the respective type of excitations (see text).

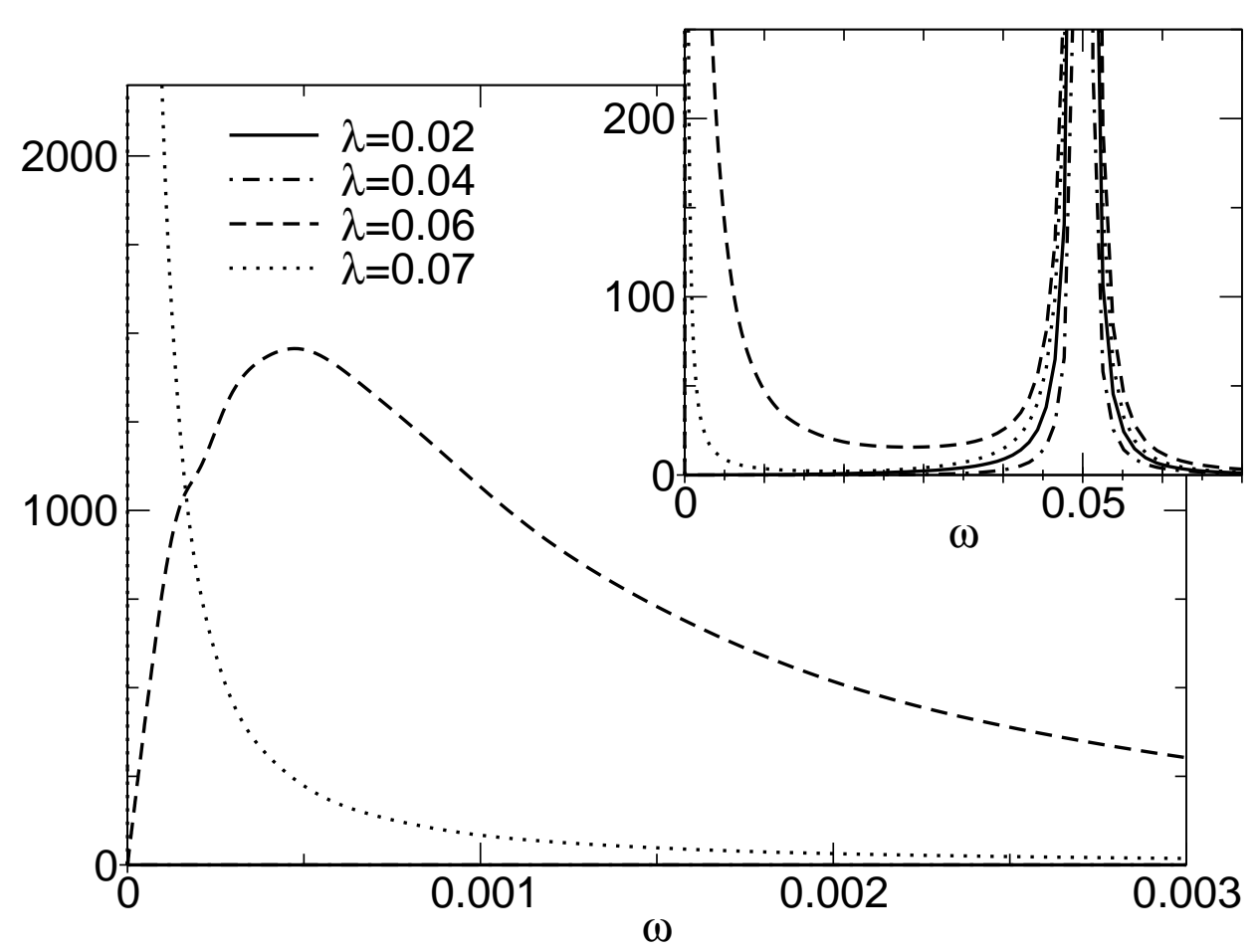

Figure 9. Imaginary part of the phonon propagator for the parameters of figure 7

in the Kondo regime and a narrow Kondo resonance at the Fermi level can clearly be distinguished from the atomic level below.

For intermediate values of $\lambda$, the spectrum again involves four dominant peaks whose relative positions and weights can be understood on the basis of the diagram on the left in figure 12. These excitations correspond exactly to the ones in equation (21). The transfer of weight with increasing $\lambda$ is rather different: in the intermediate regime, 


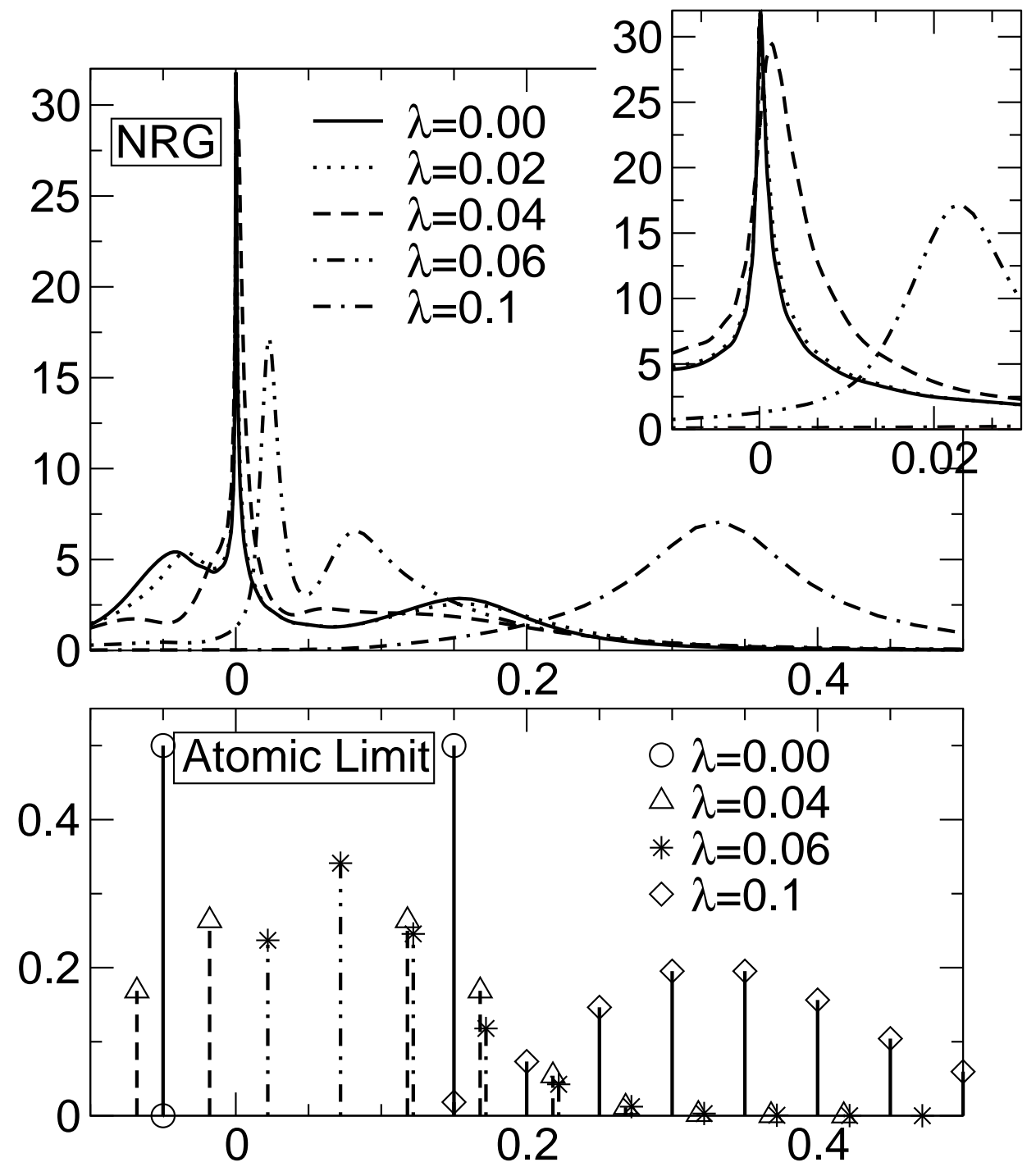

Figure 10. Same as figure 7 , but with $U=0.2$.

$0.04<\lambda<0.08$, weight is transfered from those excitations which involve an initial single-occupied state to those in which the initial state is unoccupied. As this transfer of weight develops, the Kondo resonance disappears as can be seen clearly in the inset of the top panel of figure 10 .

In the results with $U=0.05$, figure 11 and the diagram on the right in figure 12, only one peak is to be seen for $\lambda=0$. reflecting the fact that for this set of parameters, we are in the mixed-valence regime. The change in high-energy features with increase of $\lambda$ can again be understood in terms of the transfer of weights between the dominant excitations as indicated in figure 12. For large $\lambda$, the spectral weight accumulates for excitations from the double-occupied local level. Consequently, the peak moves to lower energies in a similar way to that shown in figure 5 for $U=0$ (see discussion in Sec. 4). The crossover occurs for smaller values of $\lambda$ compared to the case shown in figure 10 due to the smaller value of $U$. 


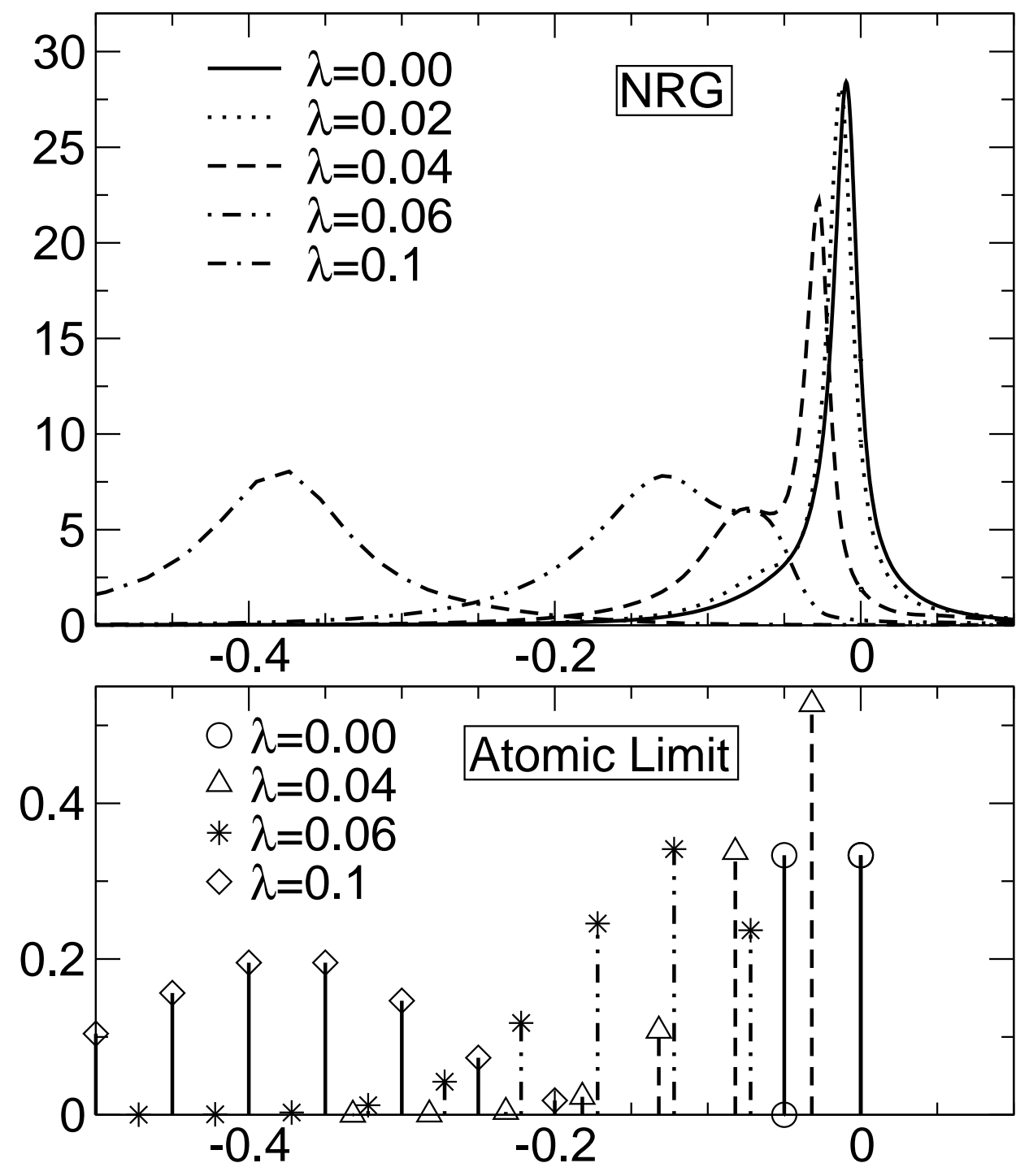

Figure 11. Same as figure \&, but with $U=0.05$.

In both these cases, the charge fluctuations are small, and there is no evidence of a soft phonon mode developing in the phonon spectrum. Also, perturbation theory does not give reasonable results for either one of these cases.

\section{Conclusions}

We begin with an overview of the detailed results presented in sections 3-6, and look first of all at the results for the symmetric model. When $\lambda=0$ and we move from $U=0$ to $U=0.1$, we move from an intermediate valence regime, in which the spin and charge fluctuations are of the same order to the Kondo regime, in which the charge fluctuations are suppressed. The central resonance narrows considerably from a width $2 \Delta$ to one determined by the Kondo temperature $T_{\mathrm{K}}$, and atomic-like high energy peaks begin to develop. When we switch on, and increase, the phonon coupling the central 

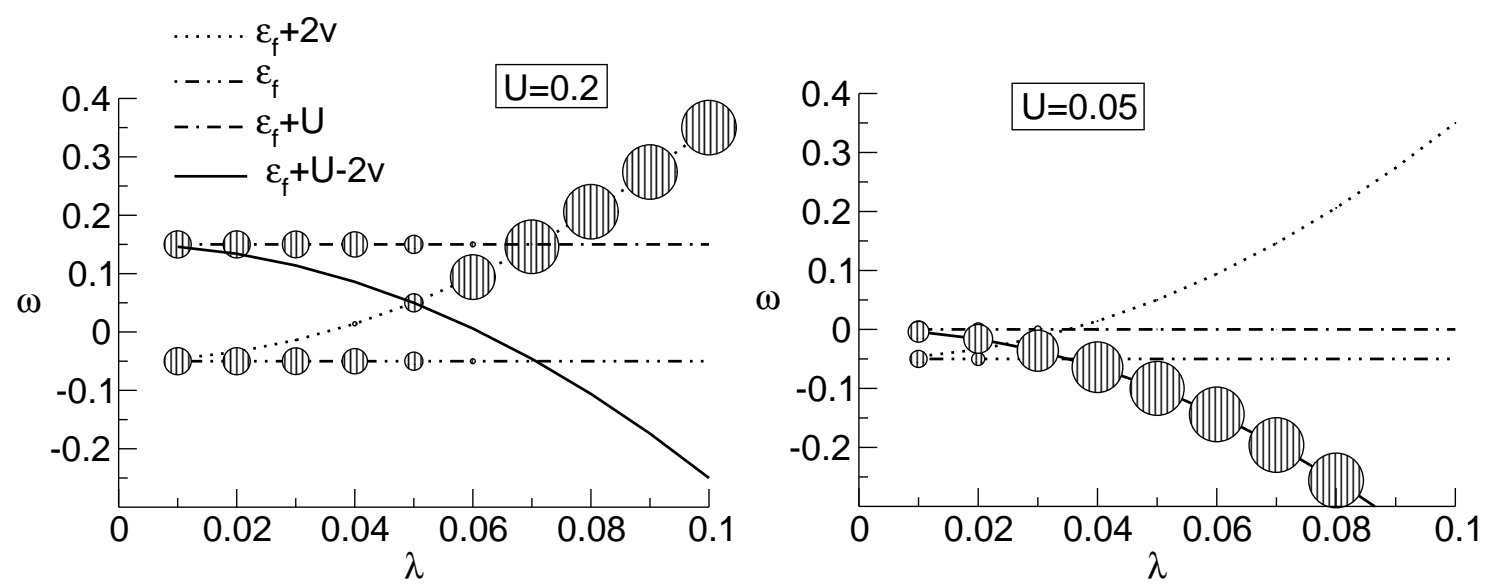

Figure 12. Positions of the dominant peaks for the atomic limit calculation from Figs. 10 and 11. The size of the circles shows the total weight for the respective type of excitations (see text).
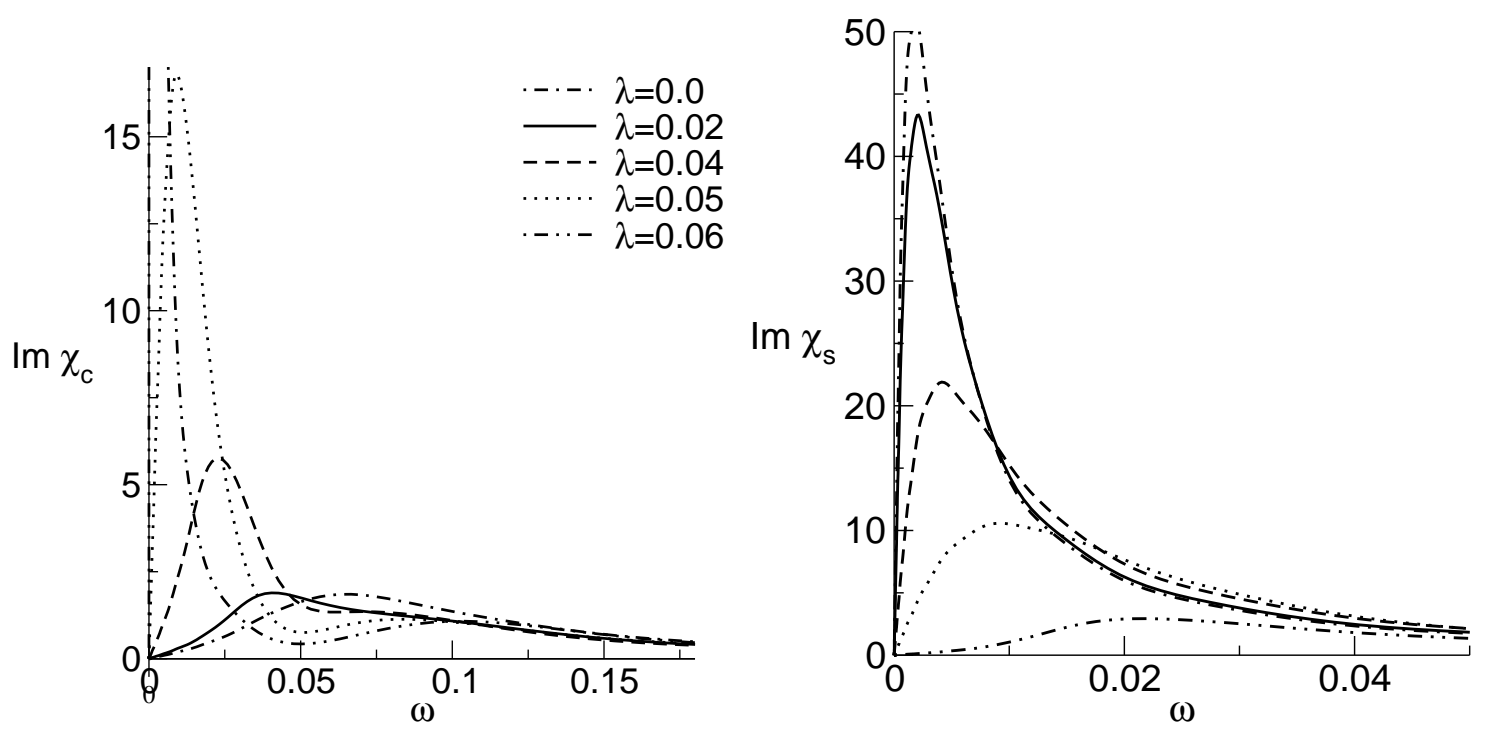

Figure 13. Imaginary part of the dynamic charge $\left(\chi_{c}\right)$ and spin susceptibilities $\left(\chi_{s}\right)$ for the symmetric parameters of figure $7\left(U=0.1, \epsilon_{f}=-0.05, V=0.1\right)$.

peak initially broadens and then for large phonon coupling strength narrows markedly. The initial broadening can be understood as mainly due to the reduction in the effective electron interaction from $U \rightarrow \bar{U}=U-2 v$, causing an increase in $T_{\mathrm{K}}$, which depends exponentially on $U$. However, the atomic-like peaks at $\omega \sim \pm U / 2$, seem to be largely unaffected. As $\bar{U}$ falls below the value $\pi \Delta$, the relative strength of the charge fluctuations increases, and we have a regime which is similar to the intermediate valence regime as the spin and charge fluctuations are of the same order, though high energy features associated with the atomic limit persist. Finally we move into the regime where $\bar{U}$ reaches zero and then becomes negative. In this limit we see that the spin fluctuations 
are largely suppressed as pairing occurs and the impurity state is either doubly-occupied or empty. Excitations from these states are associated with broadened high energy features above and below the Fermi-level. The central resonance narrows exponentially. One can interpret the narrow resonance in the strong-coupling regime as analogue of the Kondo resonance in a negative- $U$ model. In this regime the spin fluctuations have been largely eliminated but there remain long-lived charge fluctuations between the double and zero occupation states at the impurity site. The double and zero occupied states can be interpreted as the up and down configurations of a local isospin. In the $\sigma-\tau$ model an exchange coupling of such an isospin to the conduction electrons is invoked to mimic one of the channels in the two channel Kondo model [18, 19].

The reversal of roles of the spin and charge fluctuations can be seen in the results for the corresponding dynamic susceptibilities shown in figure 13 for $U=0.1$. For $\lambda=0$ there is a low energy peak in the spin susceptibility that gradually gets suppressed for increasing $\lambda$ and moves to higher frequencies. The reverse trend is seen in the charge susceptibility where a low energy peak develops and becomes very narrow in the negative $\bar{U}$ range.

We can also interpret the narrow central resonance as the zero-phonon excitation which carries very little weight in the strong-coupling regime because of the high probability of exciting a phonon when changing the impurity occupation. The narrow peak in the charge susceptibility is reflected in the phonon spectrum as low energy mode at $\omega_{\lambda}$.

Whereas the first of these interpretations would give a resonance that exponentially narrows as $e^{-|\bar{U}| / 8 \pi \Delta}$ with $\bar{U}-U \sim-\lambda^{2} / \omega_{0}$, we found that it narrows more rapidly than this expression would indicate (see figure 21). The expression for the narrowing involving the soft phonon mode, $\sim \exp \left(-v / \omega_{\lambda}\right)$ with $\omega_{\lambda} \sim \exp \left(-\lambda^{2} / \omega_{0}^{2}\right)$ gives a better fit to data.

In the results for the asymmetric model the low energy phonon mode does not develop, and there is no narrow resonance at the Fermi-level other than the original Kondo peak in the case $U=0.2$ at zero or small phonon coupling. In the large $\lambda$ regime $(\bar{U}<0)$ in the two cases studied there is either a broad peak below the Fermi-level corresponding to double occupation, or one above the Fermi-level corresponding to zero occupation. For asymmetric conditions the double and zero occupied states are not equally likely, and the local level position $\epsilon_{f}$ for $\epsilon_{f} \neq 0$ acts like a magnetic field on the isospin suppressing the 'Kondo' resonance. The same behaviour is observed in the negative- $U$ Anderson model without phonons.

Our conclusions are very much in line with those of Hewson and Newns in their study of the spinless model [13, 14]. A direct comparison with their work is not possible because no $\bar{U}$-term can be induced by the phonons in the spinless case. Their conclusion was that a significant narrowing of the local resonance could only be induced by strong phonon coupling if the impurity level lies at, or very close to the Fermi-level (intermediate valence regime), when the zero or single occupation states of the local level have the same energy, This closely resembles the situation in the model with spin, but in terms of doubly-occupied and the empty states. 
We have surveyed the physically interesting parameter regimes of the model using the NRG, and related our results to atomic limit calculations and perturbation theory. The relevant parameter regime for magnetic impurities systems is expected to be large $U$, with a phonon coupling such $v \ll U$, and $\omega_{0}<\Delta$. We have taken a relatively large value of $\omega_{0}\left(\omega_{0} \sim \pi \Delta\right)$ in order to see the features in the spectrum clearly and save computational time (see Appendix B), but results for smaller values of $\omega_{0}$ were found to be qualitatively similar.

Dynamical response functions for impurity systems are difficult to observe experimentally, so it would be interesting to calculate the effects of the phonon coupling on the thermodynamic behaviour which could be more readily compared with experiment. This we propose to do for a separate publication. As a more realistic model for magnetic impurities we also intend to include a direct coupling of the phonon mode to the hybridization term, because this term should be rather sensitive to local lattice vibrations.

Having taken the first step of calculating the dynamic properties of the impurity model with phonons, we now propose to extend these calculations to lattice models within the framework of the dynamical mean-field theory. These calculations should be particularly relevant to high- $T_{c}$ superconductors where recent photoemission experiments give evidence of strong electron-phonon coupling [20]. The electron-lattice coupling is also known to be important for the manganites [21]. The calculation could be extended to a model for these systems by including a coupling to a local spin [22], which would require only a generalization of the impurity part of the Hamiltonian. The calculation could also be extended to include a dispersive phonon mode. This would require the introduction of a second chain of states, but then only a lower proportion of states could be kept in each iteration.

\section{Acknowledgments}

We wish to thank to the EPSRC for the support of a research grant (GR/J85349), and the Newton Institute, Cambridge, where this work was initiated during their six-month

programme on 'Strongly Correlated Electron Systems'. We also thank Simon Bradley for his work in the development stage of the NRG program and Ralf Bulla for helpful discussions.

\section{Appendix A. Equations of motion}

We can use the standard equation of motion for the Fourier transform of the double-time Green's function,

$$
\langle\langle A, B\rangle\rangle_{\omega}=\left\langle[A, B]_{\eta}\right\rangle+\left\langle\left\langle[A, H]_{-}, B\right\rangle\right\rangle_{\omega}
$$


To derive the expression for the self-energy given in equation (3), we choose $A=f_{\sigma}$ and $B=f_{\sigma}^{\dagger}$ and $\eta=+$ as these are fermion operators. The equations of motion give

$$
\begin{gathered}
\left(\omega-\epsilon_{f}\right) G_{\sigma}(\omega)=1+U\left\langle\left\langle f_{\sigma} f_{\bar{\sigma}}^{\dagger} f_{\bar{\sigma}}, f_{\sigma}^{\dagger}\right\rangle\right\rangle_{\omega}+\lambda\left\langle\left\langle f_{\sigma}\left(b^{\dagger}+b\right), f_{\sigma}^{\dagger}\right\rangle\right\rangle_{\omega}+\sum_{\mathbf{k}} V_{\mathbf{k}}\left\langle\left\langle c_{\mathbf{k} \sigma}, f_{\sigma}^{\dagger}\right\rangle\right\rangle_{\omega} \\
\left(\omega-\epsilon_{\mathbf{k}}\right)\left\langle\left\langle c_{\mathbf{k} \sigma}, f_{\sigma}^{\dagger}\right\rangle\right\rangle_{\omega}=V_{\mathbf{k}} G_{\sigma}(\omega)
\end{gathered}
$$

Hence

$$
\left(\omega-\epsilon_{f}-\Delta(\omega)\right) G_{\sigma}(\omega)=1+U F_{\sigma}(\omega)+\lambda M_{\sigma}(\omega)
$$

where

$$
F_{\sigma}(\omega)=\left\langle\left\langle f_{\sigma} f_{\bar{\sigma}}^{\dagger} f_{\bar{\sigma}}, f_{\sigma}^{\dagger}\right\rangle\right\rangle_{\omega}, \quad M_{\sigma}(\omega)=\left\langle\left\langle f_{\sigma}\left(b^{\dagger}+b\right), f_{\sigma}^{\dagger}\right\rangle\right\rangle_{\omega}
$$

We define

$$
\Sigma_{\sigma}^{U}(\omega)=U \frac{F_{\sigma}(\omega)}{G_{\sigma}(\omega)}, \quad \Sigma_{\sigma}^{\lambda}(\omega)=\lambda \frac{M_{\sigma}(\omega)}{G_{\sigma}(\omega)}
$$

so that $\Sigma_{\sigma}(\omega)=\Sigma_{\sigma}^{U}(\omega)+\Sigma_{\sigma}^{\lambda}(\omega)$.

For the boson Green's function $D(\omega)$ we take $A=B=b^{\dagger}+b$ and $\eta=-$. The equation of motions of motion give

$$
\begin{aligned}
& \omega D(\omega)=\omega_{0}\left\langle\left\langle\left(-b^{\dagger}+b\right),\left(b^{\dagger}+b\right)\right\rangle\right\rangle_{\omega} \\
& \omega\left\langle\left\langle\left(-b^{\dagger}+b\right),\left(b^{\dagger}+b\right)\right\rangle\right\rangle_{\omega}=2+2 \lambda\left\langle\left\langle\left\langle\hat{Q},\left(b^{\dagger}+b\right\rangle\right\rangle_{\omega}+\omega_{0} D(\omega)\right.\right.
\end{aligned}
$$

Hence,

$$
D(\omega)=D^{0}(\omega)+\lambda D^{0}(\omega)\left\langle\left\langle\hat{O},\left(b^{\dagger}+b\right)\right\rangle\right\rangle_{\omega}
$$

where $\hat{O}=\sum_{\sigma} f_{\sigma}^{\dagger} f_{\sigma}-1$. Taking the equation of motion for the right-hand operator,

$$
\begin{aligned}
& \left.-\omega\left\langle\left\langle\hat{O},\left(b^{\dagger}+b\right)\right\rangle\right)\right\rangle_{\omega}=\omega_{0}\left\langle\left\langle\hat{O},\left(-b^{\dagger}+b\right)\right\rangle\right\rangle_{\omega} \\
& -\omega\left\langle\left\langle\hat{O},\left(-b^{\dagger}+b\right)\right\rangle\right\rangle_{\omega}=2 \lambda\langle\langle\hat{O}, \hat{O}\rangle\rangle_{\omega}+\omega_{0}\left\langle\left\langle\hat{O},\left(b^{\dagger}+b\right)\right\rangle\right\rangle_{\omega} \\
& \left\langle\left\langle\hat{O},\left(b^{\dagger}+b\right\rangle\right)\right\rangle_{\omega}=D^{0}(\omega)+\lambda D^{0}(\omega)\langle\langle\hat{O}, \hat{O}\rangle\rangle_{\omega}
\end{aligned}
$$

Hence the result,

$$
D(\omega)=D^{0}(\omega)+\lambda^{2} D^{0}(\omega)\langle\langle\hat{O}, \hat{O}\rangle\rangle_{\omega} D^{0}(\omega)
$$

\section{Appendix B. Matrix elements and NRG calculation}

To evaluate the Green's function $M_{\sigma}(\omega)$, and hence the self-energy $\Sigma_{\sigma}^{\lambda}(\omega)$, we need to calculate the matrix elements of the operator $f_{\sigma}\left(b^{\dagger}+b\right)$. We can classify the states according to total charge and total spin quantum numbers $Q, S$ and $S_{z}$, and an additional index $w$ or $r$, and we define reduced matrix elements using the Wigner-Eckart theorem in the usual way. The bose operators only enter explicitly in the calculation at the impurity site. Let the basis states used for this calculation be denoted by $\left|Q, S, S_{z}, n, r\right\rangle$ where $n$ denotes the occupation number for the bose states, and $r$ is an additional 
index. After diagonalization the exact eigenstates at the impurity site can be written in the form $\left|Q, S, S_{z}, w\right\rangle$, where $w$ no longer denotes the number of bosons, which are not conserved by the interaction term, so $w$ is a general index. We need the reduced matrix elements between these eigenstates, $\left\langle Q, S, w\left\|f_{\sigma}\left(b^{\dagger}+b\right)\right\| Q^{\prime}, S^{\prime}, w^{\prime}\right\rangle$. If

$$
\left|Q^{\prime}, S^{\prime}, w^{\prime}\right\rangle=\sum_{n^{\prime}} U_{Q^{\prime}, S^{\prime}}\left(w^{\prime}, n^{\prime} r^{\prime}\right)\left|Q^{\prime}, S^{\prime}, n^{\prime}, r^{\prime}\right\rangle
$$

then

$$
\begin{aligned}
\left\langle Q, S, w \| f_{\sigma}\left(b^{\dagger}\right.\right. & \left.+b) \| Q^{\prime}, S^{\prime}, w^{\prime}\right\rangle= \\
& =\sum_{n, n^{\prime}, r, r^{\prime}} U_{Q, S}(w, n, r) U_{Q^{\prime}, S^{\prime}}\left(w^{\prime}, n^{\prime} r^{\prime}\right)\left\langle Q, S, n, r\left\|f_{\sigma}\left(b^{\dagger}+b\right)\right\| Q^{\prime}, S^{\prime}, n^{\prime}, r^{\prime}\right\rangle \\
& =\sum_{n, r, r^{\prime}} U_{Q, S}(w, n, r)\left(\sqrt{n} U_{Q^{\prime}, S^{\prime}}\left(w^{\prime},(n-1) r^{\prime}\right)+\right. \\
& \left.+\sqrt{n+1} U_{Q^{\prime}, S^{\prime}}\left(w^{\prime},(n+1) r^{\prime}\right)\right)\left\langle Q, S\left\|f_{\sigma}\right\| Q^{\prime}, S^{\prime}\right\rangle
\end{aligned}
$$

as $\left\langle Q, S, n, r\left\|f_{\sigma}\right\| Q^{\prime}, S^{\prime}, n, r^{\prime}\right\rangle$ is independent of $n$. Hence we can deduce the values of $\left\langle Q, S, w\left\|f_{\sigma}\left(b^{\dagger}+b\right)\right\| Q^{\prime}, S^{\prime}, w^{\prime}\right\rangle$ after diagonalization at the impurity site. Once we have these initial values the equations at all later steps will take the same form as those for $\left\langle Q, S, w\left\|f_{\sigma}\right\| Q^{\prime}, S^{\prime}, w^{\prime}\right\rangle$.

The spectral density $C_{\sigma}(\omega)$ of the Green's function $M_{\sigma}(\omega)$ is given by

$C_{\sigma}(\omega)=\frac{1}{Z} \sum_{n, n^{\prime}}\left\langle n\left|\left(b+b^{\dagger}\right) f_{\sigma}\right| n^{\prime}\right\rangle\left\langle n^{\prime}\left|f_{\sigma}^{\dagger}\right| n\right\rangle \delta\left(\omega-\left(E_{n^{\prime}}-E_{n}\right)\right)\left(e^{-\beta E_{n}}+e^{-\beta E_{n^{\prime}}}\right)$

where $|n\rangle=\left|Q_{n}, S_{n}, S_{z, n}, w_{n}\right\rangle$ and $Z$ is the partition function. When expressed in terms of reduced matrix elements it takes exactly the same form as the spectral density for $G_{\sigma}$ except that the reduced matrix element $\left\langle Q, S, w\left\|f_{\sigma}\left(b^{\dagger}+b\right)\right\| Q^{\prime}, S^{\prime}, w^{\prime}\right\rangle$ replaces $\left\langle Q, S, w\left\|f_{\sigma}\right\| Q^{\prime}, S^{\prime}, w^{\prime}\right\rangle$.

\section{NRG parameters:}

The NRG calculations were performed using the discretization parameter $1.7<\Lambda<2$., and keeping at least 600 states in each iteration (see Ref. [5, 11]). The number of eigenstates of an impurity site coupled to a local phonon mode (the atomic limit (15)), is already infinite. So a cutoff of higher-energy states is already necessary at the initial step of the NRG procedure. The probability distribution function to find $n$ excited phonons in the system has a clear maximum around $n=\bar{n}=v / \omega_{0}$, as can be read off equation (15), and falls off rapidly for larger values of $n$. So in the spirit of the NRG it is sufficient to keep only states with $n<n_{\text {cutoff }}$ where we chose an initial $n_{\text {cutoff }}=4 \bar{n}$.

\section{Appendix C. Derivation of equations (8) and (9)}

Here we give details of the explicit removal of the phonon terms in the Hamiltonian to derive equations (8) and (9). We will factorise the time-ordering operator $T$ in equation 
(घ) so that $T=T_{\mathrm{F}} T_{\mathrm{B}}$, where $T_{\mathrm{F}}$ operates purely on the Fermi operators and $T_{\mathrm{B}}$ purely on the bose ones. As the interaction term with the phonons,

$$
H_{\mathrm{int}}^{\mathrm{B}}(\tau)=\lambda \hat{O}(\tau)\left(b^{\dagger} e^{\omega_{0} \tau}+b e^{-\omega_{0} \tau}\right),
$$

is linear in $b$ and $b^{\dagger}$, we can apply the well-known Baker-Hausdorff formula to deduce

$$
T_{\mathrm{F}} T_{\mathrm{B}} e^{-\int_{0}^{\beta} H_{\mathrm{int}}(\tau) d \tau}=T_{\mathrm{F}} e^{-\int_{0}^{\beta} H_{\mathrm{int}}(\tau) d \tau} e^{\frac{1}{2} \int_{0}^{\beta} \int_{0}^{\tau}\left[H_{\mathrm{int}}(\tau), H_{\mathrm{int}}\left(\tau^{\prime}\right)\right] d \tau d \tau^{\prime}} .
$$

We now take the expectation value of $e^{-\int_{0}^{\beta} H_{\text {int }}(\tau) d \tau}$ with respect to the boson states using $\left\langle e^{A}\right\rangle=e^{\frac{1}{2}\left\langle A^{2}\right\rangle}$, applicable when $A$ is a linear combination of $b$ and $b^{\dagger}$ [17]. We find in the exponential,

$$
\frac{\lambda^{2}}{2} \int_{0}^{\beta} \int_{0}^{\tau} \hat{O}(\tau) \hat{O}\left(\tau^{\prime}\right)\left(\gamma\left(\tau-\tau^{\prime}\right)+\gamma\left(\tau^{\prime}-\tau\right)\right) d \tau d \tau^{\prime}-\int_{0}^{\beta} H_{\mathrm{int}}^{\mathrm{F}}(\tau) d \tau,
$$

where $H_{\text {int }}^{\mathrm{F}}(\tau)$ is the purely fermion interaction term, and

$$
\gamma\left(\tau-\tau^{\prime}\right)=n\left(\omega_{0}\right) e^{\omega_{0}\left(\tau-\tau^{\prime}\right)}+\left(1+n\left(\omega_{0}\right)\right) e^{-\omega_{0}\left(\tau-\tau^{\prime}\right)} .
$$

We also have that

$$
\left[H_{\mathrm{int}}(\tau), H_{\mathrm{int}}\left(\tau^{\prime}\right)\right]=\frac{\lambda^{2}}{2} \hat{O}(\tau) \hat{O}\left(\tau^{\prime}\right)\left(e^{-\omega_{0}\left(\tau-\tau^{\prime}\right)}-e^{\omega_{0}\left(\tau-\tau^{\prime}\right)}\right),
$$

subject to the fermion time-ordering within the complete expression.

Finally, we find

$$
Z / Z_{0}=\left\langle T_{\mathrm{F}} e^{-\int_{0}^{\beta} H_{\mathrm{int}}^{\mathrm{F}}(\tau) d \tau} e^{-\lambda^{2} \int_{0}^{\beta} \int_{0}^{\tau} \hat{O}(\tau) \hat{O}\left(\tau^{\prime}\right) D^{0}\left(\tau-\tau^{\prime}\right) d \tau d \tau^{\prime}}\right\rangle_{\mathrm{F}}
$$

where

$$
D^{0}\left(\tau-\tau^{\prime}\right)=\left(\frac{e^{-\omega_{0}\left|\tau-\tau^{\prime}\right|}}{1-e^{-\omega_{0} \beta}}+\frac{e^{\omega_{0}\left|\tau-\tau^{\prime}\right|}}{e^{\omega_{0} \beta}-1}\right),
$$

which is the imaginary time representation of the phonon Green's function.

\section{References}

[1] T. Holstein, Ann. Phys. 8, 3251959.

[2] A. Georges, G. Kotliar, W. Krauth, and M. J. Rozenberg, Rev. Mod. Phys. 68(1), 131996.

[3] W. Metzner and D. Vollhardt, Phys. Rev. Lett. 62, 3241989.

[4] K. Wilson, Rev. Mod. Phys. 47(4), 7731975.

[5] H. R. Krishna-murthy, J. W. Wilkins, and K. G. Wilson, Phys. Rev. B 21(3), 10031980.

[6] H. R. Krishna-murthy, J. W. Wilkins, and K. G. Wilson, Phys. Rev. B 21(3), 10441980.

[7] O. Sakai, Y. Shimizu, and T. Kasuya, J. Phys. Soc. Japan 58(10), 36661989.

[8] T. A. Costi, A. Hewson, and V. Zlatic, J. Phys.: Condens. Matter 6, 25191994.

[9] O. Sakai and Y. Kuramoto, Solid State Commun. 89, 3071994.

[10] R. Bulla, Phys. Rev. Lett. 83, 1361999.

[11] R. Bulla, T. Costi, and D. Vollhardt, Phys. Rev. B 64, 0451032001.

[12] P. W. Anderson, Phys. Rev. 124(1), 411961.

[13] A. C. Hewson and D. M. Newns, J. Phys. C 12, 16651979.

[14] A. C. Hewson and D. M. Newns, J. Phys. C 13, 44771980.

[15] R. Bulla, A. C. Hewson, and T. Pruschke, J. Phys.: Condens. Matter 10, 83651998.

[16] J. W. Negele and H. Orland, Quantum Many-Particle systems, Addison-Wesley 1988.

[17] G. D. Mahan, Many-Particle Physics, Plenum Press 1990. 
[18] P. Coleman, L. Ioffe, and A. M. Tsvelik, Phys. Rev. B 52, 66111995.

[19] S. C. Bradley, R. Bulla, A. C. Hewson, and G.-M. Zhang, Eur. Phys. J. B 11, 5351999.

[20] A. Lanzara, P. V. Bogdanov, X. J. Zhou, S. A. Keller, D. L. Feng, E. D. Lu, T. Yoshida, H. Eisaki, A. Fujimori, K. Kishio, J.-I. Shimoyama, T. Nodaand S. Uchida, Z. Hussain, and Z.-X. Shen, Nature 412, 5102001.

[21] A. P. Ramirez, J. Phys.: Condens. Matter 9, 81711997.

[22] A. J. Millis, P. B. Littlewood, and B. I. Shraiman, Phys. Rev. Lett. 74(25), 51441995. 